\title{
Numerical modeling of the debris flows runout
}

\author{
Francesco Federico ${ }^{1, *}$, and Chiara Cesali $^{1}$ \\ ${ }^{1}$ University of Rome 'Tor Vergata', Department of Civil and Information Engineering, Via del Politecnico 1, 00133 Rome, Italy
}

\begin{abstract}
Rapid debris flows are identified among the most dangerous of all landslides. Due to their destructive potential, the runout length has to be predicted to define the hazardous areas and design safeguarding measures. To this purpose, a continuum model to predict the debris flows mobility is developed. It is based on the well known depth-integrated avalanche model proposed by Savage and Hutter ( $S \& H$ model) to simulate the dry granular materials flows. Conservation of mass and momentum equations, describing the evolving geometry and the depth averaged velocity distribution, are re-written taking into account the effects of the interstitial pressures and the possible variation of mass along the motion due to erosion/deposition processes. Furthermore, the mechanical behaviour of the debris flow is described by a recently developed rheological law, which allows to take into account the dissipative effects of the grain inelastic collisions and friction, simultaneously acting within a 'shear layer', typically at the base of the debris flows. The governing PDEs are solved by applying the finite difference method. The analysis of a documented case is finally carried out.
\end{abstract}

\section{Introduction}

Savage and Hutter (1989) applied for the first time the depth averaged Saint Venant equations to analyze the propagation of sliding granular masses along an inclined planar surface [1].

By considering the $x$-coordinate in the downflow direction and the $z$-coordinate in the normal direction to the sliding surface and assuming that $(i)$ the downslope velocity is constant along the depth $(v=v(x, t))$; (ii) the density $\rho$ of the granular material is constant; (iii) the granular material is dry; (iv) the mass is constant; (v) the granular material obeys the Mohr-Coulomb resistance law within the deforming mass (with internal friction angle $\phi$ ) and along the sliding surface (with basal friction angle $\delta$ ), by referring to an element of length $d x$ (Fig. 1), the following mass balance and $x$-momentum balance equations may be written [2]:

$$
\begin{gathered}
\frac{\partial H}{\partial t}+\frac{\partial}{\partial x}(v \cdot H)=0 \\
\frac{\partial v}{\partial t}+v \frac{\partial v}{\partial x}=g \sin \theta-\operatorname{sgn}(v) g \tan \delta(\cos \theta)-g K_{a c t, p a s} \cos \theta \frac{\partial H}{\partial x}
\end{gathered}
$$

$H(x, t)$ is the thickness or depth of the sliding granular mass; $v(x, t)$ is the $x$-component of the velocity vector; $g$ is the gravity acceleration; $\theta$ is the slope of the sliding surface; $K_{a c t \text {,pas }}$ is the active (if $\partial v / \partial x \geq 0$ ) or passive (if $\partial v / \partial x<0)$ earth pressure coefficient:

$$
K_{a c t, p a s}=2 \cdot\left[\left(1 \pm \sqrt{1-\cos ^{2} \phi\left(1+\tan ^{2} \delta\right)}\right) / \cos ^{2} \phi\right]-1
$$

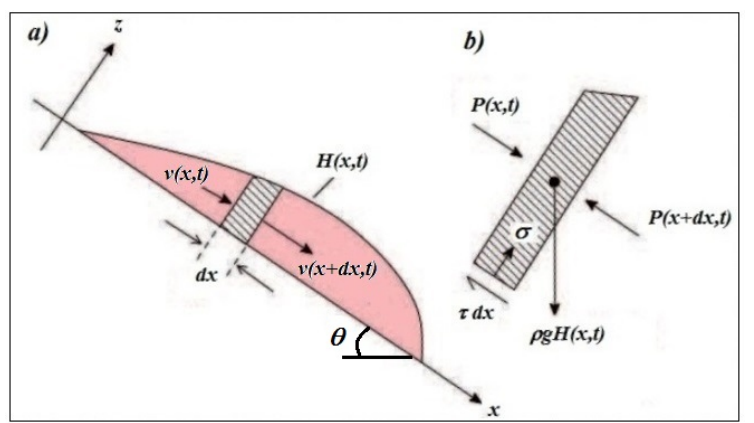

Fig. 1. Granular mass sliding along an inclined planar surface: a) geometry and coordinate system; $b$ ) forces acting on an infinitesimal element.

\section{Proposed model}

Equations (1) and (2) are rewritten by taking into account the effects of the pore water pressures and the variation of mass due to erosion or deposition processes.

Moreover, the curvature of the sliding surface and the typical (frictional-collisional) mechanical behaviour of a debris flow [3] are considered.

\#

\subsection{Curvature of the sliding surface}

If the sliding surface is geometrically described by an arc of circumference (curvature $k \neq 0$ ), the centrifugal force occurs; this force modifies the normal resultant force ( $\sigma$ $d x)$ and, then, the shear resistance force $(\tau d x)$ :

$$
\tau \cdot d x=-\operatorname{sgn}(v) \cdot \rho H \cdot\left(g \cos \theta+k v^{2}\right) \tan \delta \cdot d x
$$

$\rho$ being the mass density per unit volume. 


\subsection{Pore water pressure}

Pore water pressure $\left(p_{w}\right)$ induces the reduction of the normal resultant force and modifies the lateral pressures $\left(p_{L}\right)$. Furthermore, if the curvature $k$ of the sliding surface assumes high values, $p_{w}$ doesn't vary hydrostatically [4]: a change of the direction of motion of a fluid particle (curvilinear path) implies an increase of piezometric head along the direction normal to the sliding surface. Thus, the pore water pressure at the base $\left(p_{w, b}\right)$ of the sliding mass is expressed as follows:

$$
p_{w, b}=\gamma_{w} H_{w}\left(\cos \theta+k \frac{v^{2}}{g}\right)
$$

$\gamma_{w}$ being the specific weight of water and $H_{w}$ the elevation of phreatic surface on the slope bed (Fig. 2). Additional excess pore water pressures due to changes of total stresses under undrained conditions, typically taking place within fine-grained materials as shown in centrifuge tests [5], are herein neglected. Moreover, the dissipation of excess pore water pressures due to consolidation processes is not taken into account [6].

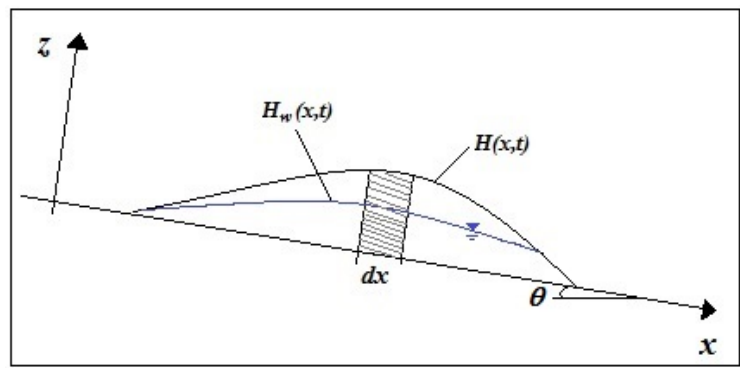

Fig. 2. Saturated or partially saturated sliding granular mass: definition of $H_{w}$.

According to (5), the shear resistance force becomes:

$$
\tau \cdot d x=-\operatorname{sgn}(v) \cdot\left(\rho g H-H_{w} \gamma_{w}\right) \cdot\left(\cos \theta+k \frac{v^{2}}{g}\right) \tan \delta \cdot d x
$$

and the resultant $P(=P(x, t)-P(x+d x, t)$, Fig. 1$)$ of the lateral pressures $\left(p_{L}\right)$ is expressed as follows:

$$
\begin{aligned}
& P=-\int_{0}^{H} \frac{\partial p_{L}}{\partial x} d z d x=-\left[K_{\text {act, pass }} \cdot\left(\cos \theta+k \frac{v^{2}}{g}\right) .\right. \\
& \left.\left(\rho g H \frac{\partial H}{\partial x}-\gamma_{w} H_{w} \frac{\partial H_{w}}{\partial x}\right)+\gamma_{w} H_{w} \frac{\partial H_{w}}{\partial x}\left(\cos \theta+k \frac{v^{2}}{g}\right)\right] d x
\end{aligned}
$$

\subsection{Mass variation}

The mass of a debris flow may change along the motion due to erosion or deposition processes [7]. Typically, the erosion phenomenon mainly occurs at high elevation, induced by the high slope and the great travel speed, while the deposition, caused by the slowdown due in turn to the reduction of slope, downstream occurs. The erosion (or deposition) process is considered by introducing the erosion (or deposition) rate $\varepsilon(v)$, dependent on the velocity of the sliding mass. It is simply assumed: $\varepsilon(v)=\varepsilon_{e r}$, if $v \geq v_{\text {crit }} ; \varepsilon(v)=\varepsilon_{\text {dep }}$, if $v<$ $v_{\text {crit }} \cdot \varepsilon_{\text {er }}(>0$, constant $)$ is the erosion rate; $\varepsilon_{\text {dep }}(<0$, constant) is the deposition rate; $v_{\text {crit }}$ is the critical velocity for which the transition from erosion to deposition process occurs. Generally, $v_{\text {crit }}$ corresponds to a limit angle of erosion, $\beta_{e}$, evaluable through the relation $[8]$ :

$$
\beta_{e}=\arctan \left\{\tan \delta\left[1-2 \frac{\rho_{w}}{\rho_{\text {bed }}}\left(1+\frac{\rho}{\rho_{\text {bed }}}\right)^{-1}\right]\right\}
$$

where $\rho_{w}$ is the density of water and $\rho_{b e d}$ is the density of the material lying on the bed of the channel (typically, $\left.1800-2000 \mathrm{~kg} / \mathrm{m}^{3}\right)$.

\subsection{Frictional-collisional mechanical behaviour}

Experimental observations showed the growth of a "shear layer" at the base of a sliding granular mass [9], where initially great deformations and then dilation and collisions between solid grains occur, differently from the top, causing a fluidification effect [10] coupled with energy dissipations. Thus, in a rapid sliding granular mass, normal and shear stresses, associated with frictional and collisional regimes, respectively, simultaneously occur [11].

Since a rapidly debris flowing is affected by a 'mixed' frictional - collisional mechanical behaviour, the shear resistance (resultant) force may be approximately expressed as $[3,12]$ :

$$
\tau \cdot d x=-\operatorname{sgn}(v)\left(\bar{r}^{-} \cdot \tau_{f r}+r \cdot \tau_{\text {disp }}\right) \cdot d x
$$

where $\tau_{f r}$ is the shear stress associated with frictional regime and $\tau_{\text {disp }}$ is the shear stress associated with collisional regime. For the normal to the boundary and shear components of the collisional (dispersive) stresses (pressures), the following relationships are respectively proposed [13]:

$$
p_{\text {disp }}=a_{i} \rho_{s} \lambda^{2} d_{p}^{2} \cos \phi\left(\frac{\partial u}{\partial z}\right)^{2} ; \tau_{\text {disp }}=a_{i} \rho_{s} \lambda^{2} d_{p}^{2} \sin \phi\left(\frac{\partial u}{\partial z}\right)^{2}
$$

$a_{\mathrm{i}}$ being the "Bagnold coefficient" (suggested value $0.042) ; \rho_{s}$, the solid fraction mass density $(2300 \div 2800$ $\left.\mathrm{kg} / \mathrm{m}^{3}\right) ; \lambda$, the "linear concentration", dependent on the solid fraction $v_{\mathrm{s}}\left(\lambda=1 /\left[\left(v_{\max } / v_{\mathrm{s}}\right)^{1 / 3}-1\right], v_{\max }(\operatorname{maximum}\right.$ solid fraction) $=0.74$ for spherical grains $) ; d_{\mathrm{p}}$ is the average grain diameter.

According to Mohr-Coulomb resistance law, the frictional shear stress $\tau_{f r}$ is thus expressed as follows:

$$
\tau_{f r}=\left[\left(\rho H-\rho_{w} H_{w}\right) \cdot\left(g \cos \theta+k v^{2}\right)+p_{d i s p}\right] \tan \delta
$$

$r$ and $\bar{r}$ (defined as $1-r$ ), dependent on the velocity $v$, allow a "weighted" balance of the shear resultant force between the frictional and dispersive stresses, statistically acting along the irregular sliding surface. It is proposed [3]: 


$$
r(v)=\frac{1}{\pi}\left\{\arctan \left[\eta\left(v-U_{c r}\right)\right]-\arctan \left[-\eta\left(v+U_{c r}\right)\right]\right\}
$$

$\eta$ is a parameter falling in the range $0.005 \div 0.5$ [3], which modulates the shape of $r ; U_{c r}$ is the critical speed for which the regime dominated by the inertial forces becomes a collisional regime: $U_{c r}=\left[\left(\rho_{s}\right.\right.$ $\left.\left.\rho_{w}\right) \cdot N_{s a v} b_{\mathrm{s}}^{2} g H_{0} /\left(\rho_{s} \cdot d_{p}^{2}\right)\right]^{1 / 2}[14]$, where $N_{\text {sav }}$ is the Savage number equal to $0.1, b_{\mathrm{s}}$ is the average shear layer thickness $\left(=10 \div 15 \cdot d_{\mathrm{p}}\right)[15], H_{0}$ is the maximum initial thickness of the granular mass. If a linear change of velocity $v$ along $z$ within the shear layer is assumed, $p_{\text {disp }}$ and $\tau_{\text {disp }}$ become proportional to $v^{2}$.

\subsection{Fundamental equations}

Under the previous considerations, the mass balance and $x$-momentum balance equations are rewritten as follows:

$$
\begin{gathered}
\frac{\partial H}{\partial t}+\frac{\partial}{\partial x}(v \cdot H)-\varepsilon(v)=0 \\
\frac{\partial v}{\partial t}+v \frac{\partial v}{\partial x}=g \sin \theta-\operatorname{sgn}(v) \cdot-\dot{r} \cdot\left[\left(1-R_{u}\right) \cdot\left(g \cos \theta+k v^{2}\right)+\right. \\
\left.\left.\left(p_{d i s p} / \rho H\right)\right] \tan \delta+r \cdot\left(\tau_{d i s p} / \rho H\right)\right\}-\left[\left(1-R_{u} \cdot d_{w}\right) K_{\text {act, pass }}+\right. \\
\left.R_{u} \cdot d_{w}\right] \cdot\left(g \cos \theta+k v^{2}\right) \frac{\partial H}{\partial x}
\end{gathered}
$$

where $d_{w}$ and $R_{u}$ are respectively defined as $d_{w}=H_{w} / H$ and $R_{u}=\gamma_{w} H_{w} /(\gamma H)=\left(\gamma_{w} / \gamma\right) \cdot d_{w}$. The coefficient $R_{u}$ can assume the following values: $R_{u}=0$, if $d_{w}=0$ (dry material); $R_{u} \in\left(0, \gamma_{w} / \gamma\right)$, if $d_{w} \in(0,1) ; R_{u}=\gamma_{w} / \gamma$, if $d_{w}=1$ (saturated flow); $R_{u} \in\left(\gamma_{w} / \gamma, 1\right)$, if $d_{w} \in\left(1, \gamma / \gamma_{w}\right) ; R_{u}=1$, if $d_{w}=\gamma / \gamma_{w} . d_{w} \in\left(1, \gamma / \gamma_{w}\right)$ roughly allows to simulate the excess pore water pressure at the base of high speed coarse-grained materials flows, due to the rapid change of pores volume related to the continuous rearrangement of grains, localized within the shear layer [16]. $R_{u}=1$ implies that the pore water pressure $\left(p_{w}\right)$ equals the total normal stress $(\sigma)$ and the frictional strength of the granular material is null. The system of governing PDEs (eqs. (14), (15)) is numerically solved.

\section{Discretization of governing PDEs}

The numerical solution of the system of governing PDEs is obtained by applying the Finite Difference Method (FDM). To this purpose, the granular mass profile is divided in $N$ cells (Fig. 3$)$ : the index $j(=0,1 \ldots N)$ corresponds to the cell boundary points. The mass balance equation (14) is discretized according to $H \& S$ formulation (first order approximation) [2]:

$$
H_{j}^{t}=\left(H_{j}^{t-1}+\varepsilon \cdot \Delta t\right) \cdot \frac{\left(x_{j+1}^{t-1}-x_{j}^{t-1}\right)}{\left(x_{j+1}^{t}-x_{j}^{t}\right)}
$$

with $x_{j}^{t}=x_{j}^{t-1}+u_{j}^{t-1} \cdot \Delta t(\Delta t=$ integration time interval $)$. The $x$-momentum balance equation (15) is discretized according to an implicit method.

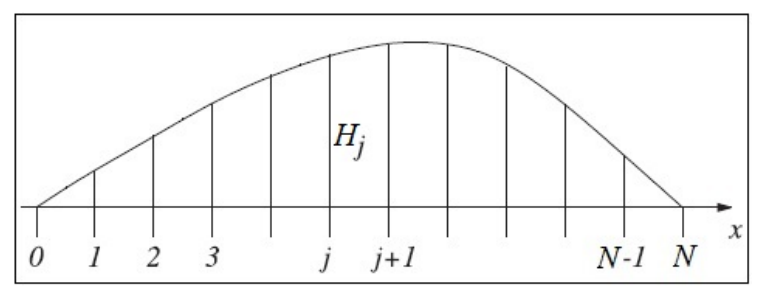

Fig. 3. Definition of mesh cell notation.

In particular, for the intermediate cells (except the first and the last two ones), $\partial H / \partial x$ (and $\partial v / \partial x$ ) is replaced by the following fourth order approximate expression:

$$
\frac{\partial H}{\partial x}=\frac{H_{j-2}^{t}-8 H_{j-1}^{t}+8 H_{j+1}^{t}-H_{j+2}^{t}}{12 \Delta x}
$$

( $\Delta x=$ integration space interval) and $\partial v / \partial t$ is replaced by its second order approximation:

$$
\frac{\partial v}{\partial t}=\frac{1}{\Delta t} \cdot\left[v_{j}^{t}-\frac{1}{2}\left(v_{j+1}^{t-1}+v_{j-1}^{t-1}\right)\right]
$$

For the first and the last cells, the spatial $(\partial H / \partial x$, $\partial v / \partial x)$ and temporal $(\partial v / \partial t)$ derivatives are replaced by the second order 'one side' and first order approximations, respectively:

$$
\frac{\partial H}{\partial x}=\frac{\mp 3 H_{j}^{t} \pm 4 H_{j \pm 1}^{t} \mp H_{j \pm 2}^{t}}{2 \Delta x} ; \frac{\partial v}{\partial t}=\frac{v_{j}^{t}-v_{j}^{t-1}}{\Delta t}
$$

For the second and the second-last cells, $\partial H / \partial x$ (and $\partial v / \partial x)$ is replaced by the following approximation:

$$
\frac{\partial H}{\partial x}=\frac{H_{j+1}^{t}-H^{t}{ }_{j-1}}{2 \Delta x}
$$

and $\partial v / \partial t$ is replaced by eq. (18). The numerical scheme proposed to solve the system of governing PDEs is implemented through a MATLAB iterative code.

In order to prevent the blow-up of the numerical solution, the CFL condition is imposed; in particular, $v_{j}^{t}$. $\Delta t / L_{j}^{t}<1$, where $L_{j}^{t}\left(=x_{j+1}{ }^{t}-x_{j}^{t}\right)$ is the width of the $i$-th cell. If this condition is not fulfilled, smaller values of $\Delta t$ must be chosen, also taking into account the computational time for the simulation.

The iterative procedure of generation of the solution of the system of governing PDEs $\left(H_{j}^{t}, v_{j}^{t}\right)$ definitively stops if during deceleration of the sliding mass: $v_{m}{ }^{t}<t o l$, where $v_{m}{ }^{t}$ is the average velocity of the cells (or debris flow) and tol is the speed tolerance.

Discretization variables (e.g. $N, \Delta t$ ) as well as the role of the additional parameters related to the collisional behaviour [3] have been parametrically evaluated.

\section{Case study: Champlong landslide}

The Champlong landslide occurred in the Val d'Aosta on October 2000. The failure surface developed within a moraine cover, inducing the detachment of a volume of approximately $10^{4} \mathrm{~m}^{3}$. The debris flow involved the 
deposits lying on the bed of the channel (width $40 \mathrm{~m}$ ), increasing its volume. The maximum moved volume, the traveled distance (projected on horizontal plane) and the maximum velocity are estimated at approximately $2 \cdot 10^{4}$ $\mathrm{m}^{3}, 870 \mathrm{~m}, 15 \mathrm{~m} / \mathrm{s}$, respectively [17]. To apply the proposed model, the sliding surface is schematized (Fig. 4) by two planar surfaces $\left(\theta_{l}=27^{\circ}\right.$ and length $=500 \mathrm{~m}, \theta_{2}$ $\left.=14^{\circ}\right)$ linked by an arc of circumference $(k=1 / 1000 \mathrm{~m})$.

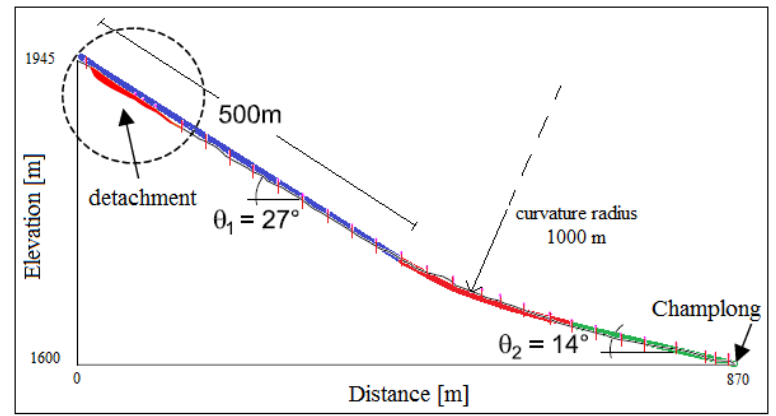

Fig. 4. Champlong landslide: path profile.

The initial (detached) mass is represented by a block (parallelepipedal shape) of thickness $H_{0}=2 \mathrm{~m}$ and width/length $L=125 \mathrm{~m}$; the following values for the remaining input parameters are selected: $\phi=40^{\circ}$; $d_{w}=0.7 ; \delta=23^{\circ} ; N=20 ; \Delta t=0.01 \mathrm{~s} ; \rho_{\mathrm{s}}=2500 \mathrm{~kg} / \mathrm{m}^{3} ; v_{\mathrm{s}}$ $=0.6 ; d_{p}=0.04 \mathrm{~m} ; \eta=0.005 ; \varepsilon_{e r}=0.01 \mathrm{~m} / \mathrm{s} ; \varepsilon_{\text {dep }}=$ $0.0001 \mathrm{~m} / \mathrm{s}$. The erosion/deposition rate has been chosen in order to obtain the maximum value of the involved volume, in situ measured.

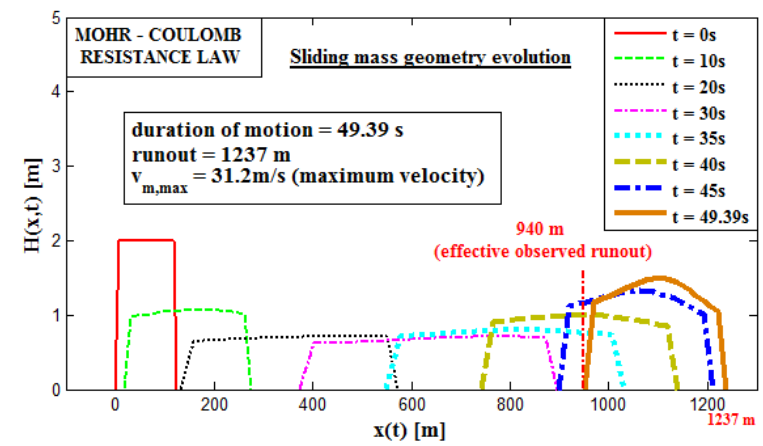

Fig. 5. $H(x, t)$ vs $x(t)$ : Mohr-Coulomb resistance law.

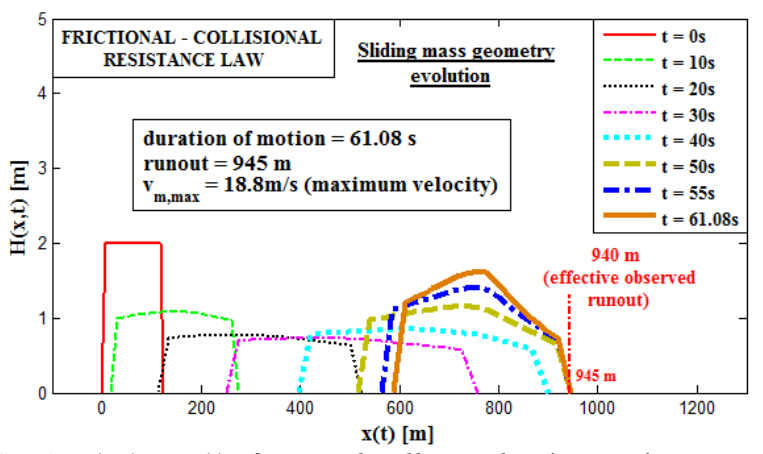

Fig. 6. $H(x, t)$ vs $x(t)$ : frictional-collisional resistance law.

The results of simulations, in terms of sliding mass geometry evolution, computed according to MohrCoulomb and frictional-collisional resistance laws are shown in Figs. 5, 6. The maximum velocity $(31.2 \mathrm{~m} / \mathrm{s})$ and runout length $(1237 \mathrm{~m})$ computed according to
Mohr-Coulomb resistance law appear remarkably greater than the corresponding values $(18.8 \mathrm{~m} / \mathrm{s}, 945 \mathrm{~m})$ obtained according to frictional-collisional resistance law, close to those ones in situ observed $(15 \mathrm{~m} / \mathrm{s}, 940 \mathrm{~m})$.

\section{Concluding remarks}

Based on the well known $S \& H$ model, a continuum model to predict the debris flows mobility is proposed. It allows to take into account the effects of the interstitial pressures and the possible variation of mass along the motion due to erosion/deposition processes as well as the typical (frictional-collisional) mechanical behaviour of a debris flow, affected by grain inelastic collisions and friction, simultaneously acting within a basal 'shear layer', causing complex dissipative effects.

The analysis of the Champlong landslide showed that, through a suitable choice of the input parameters, the proposed model allows to produce the in situ observed runout. Particularly, the comparison with the results obtained by assuming the conventional purely frictional resistance law (Mohr-Coulomb) pointed out the key role played by the additional input parameters related to the collisional behaviour (e.g. $d_{p}$, the average grain diameter; $\eta$, the modulator parameter; $v_{\mathrm{s}}$, the solid fraction of the shear layer) on the final runout length.

\section{References}

1. S.B. Savage, K. Hutter, J. Fluid Mech. 199, 177 215 (1989)

2. S.P. Pudasaini, K. Hutter, Avalanche dynamics. Dynamics of rapid flows of dense granular avalanches (Springer-Verlag Berlin, 2007)

3. F. Federico, C. Cesali, Can. Geotech. J. 52, 1 - 21 (2015)

4. G. De Marchi, Idraulica. Basi scientifiche e applicazioni tecniche (Ulrico Hoepli, Milano, 1961)

5. A.F. Rauch, K. Ladwig, P.J. Amaya, World of Coal Ash Conference (Nasvhille, Ash Library, 2015)

6. J.N. Hutchinson, Can. Geotech. J. 23, 115 - 126 (1986)

7. O. Hungr, Int. W. on Occurrence and Mechanisms of Flow-Like Landslides in Natural Slopes and Earthfills (Kluwer Publishers, Sorrento, 2004)

8. T. Takahashi, Debris Flow (IAHR Monograph Series, Balkema Publishers, Rotterdam, 1991)

9. O. Hungr, Can. Geotech. J. 32, 610 - 623 (1995)

10. O. Hungr, S.G. Evans, 7th Int. Symp. on Landslides 1, 233 - 238 (Trondheim, 1996)

11. D. Zhang, M.A. Foda, Ac. Mech. 121, 1 - 19 (1997)

12. A. Armanini, J. of Hydraulic Research 51 (2), $111-$ 120 (2013)

13. R. A. Bagnold, P.R.S. London 225, 49 - 63 (1954)

14. R.M. Iverson, The physics of debris flows (American Geophysical Union, 1997)

15. S. Straub, Geol. Rundschau 86, 415 - 425 (1997)

16. A. Musso, F. Federico, G. Troiano, Comp. and Geotech. 31, 209 - 226 (2004)

17. M. Rosso, C. Cavallo, R. Sesenna, E. Citera, M. Cussotto, GE.A.M. 110, 61 - 69 (2003) 